bus Hodgkin, Brustkrebs und Hodenkrebs. Bei bestimmten, wenn auch seltenen bösartigen Erkrankungen wie dem Li-Fraumeni-Syndrom oder einem Retinoblastom empfiehlt es sich, wegen des extrem erhöhten Risikos für Zweittumoren auf eine Strahlentherapie zu verzichten, erklärte Reinacher-Schick.

Eine Chemotherapie mit alkylierenden Substanzen wie Cyclophosphamid erhöht die Gefahr einer Leukämie nach einer Latenz von zehn Jahren. Auch für Topoisomerasehemmer oder Platinderivate ist das Risiko erhöht. Bislang noch unklar ist, ob dies auch für Rituximab, eine Hochdosis-Chemotherapie oder eine autologe Stammzelltransplantation der Fall ist.
Um das individuelle Risiko eines $\mathrm{Pa}$ tienten für Zweittumoren abzuschätzen, sollte man sich als behandelnder Arzt nach Familiengeschichte und Vorbehandlung erkundigen. Auch Faktoren wie BMI und Rauchen müssen berücksichtigt werden. „Vor allem bei genetischer Disposition ist das Zweittumorrisiko extrem hoch “, erklärte ReinacherSchick.

\section{Was kann der Patient selbst tun?}

Der Patient selbst kann präventiv tätig werden, indem er sein Gewicht reduziert, was insbesondere das Brustkrebsrisiko positiv beeinflusst. Mehr Bewegung (etwa 30 Minuten/Tag) vermindert das Krebsrisiko ebenso, vor allem aber eine konsequente Nikotinabstinenz. Chemopräventiv kommen nur Tamoxifen und Aromatasehemmer bei Brustkrebs in Frage.

Insgesamt plädierte ReinacherSchick für eine bessere Nachsorge von Krebspatienten. Das bedeutet, die entsprechenden Kontrolluntersuchungen über fünf Jahre hinaus auszudehnen, auf häufige Tumoren zu screenen und genetische Ursachen zu erkennen.

Martin Bischoff .

- Klinisches Symposium „Der geheilte Krebspatient" im Rahmen des 119. Kongresses der Deutschen Gesellschaft für Innere Medizin e. V. am 6. April 2013 in Wiesbaden

\title{
Die Spätfolgen sind erheblich
}

— In Deutschland erkranken jedes Jahr 2500 Kinder und Jugendliche neu an Krebs. Etwa die Hälfte dieser Krebserkrankungen sind Leukämien und Lymphome, gefolgt von der großen Gruppe der Hirntumoren mit 19\%. Im weiteren handelt es sich um Neuroblastome, Gewebstumoren, Weichteiltumoren und andere. Karzinome finden sich im Kindesalter mit Ausnahme der Schilddrüsenkarzinome praktisch nicht, erklärte Prof. Dr. Charlotte Niemeyer vom Universitätsklinikum Freiburg. Krebs ist nach Unfällen die zweithäufigste Todesursache jenseits des ersten Lebensjahres.

\section{Physische und psychische Folgen}

„Aber $80 \%$ der Kinder und Jugendlichen überleben heute ihre Krebserkrankung," berichtete die Kinderonkologin. Damit ist einer von 250 jungen Menschen in Deutschland ein Überlebender einer solchen Erkrankung. Die Wahrscheinlichkeit, im weiteren Leben ein Sekundärmalignom, andere sekundäre Organschäden oder psychische Belastungssituationen zu entwickeln, ist beträchtlich.

Sehr häufig werden diese jungen Menschen mit kardialen Spätfolgen kon- frontiert: Kardiomyopathien, Klappenfehler, Perikarditis, Perikard-Fibrose, aber auch KHK und Herzrhythmusstörungen. Histologisch lässt sich erkennen, ob es sich primär um die Folge einer Strahlentherapie oder von Anthrazyklinen handelt.

Weitere Organmanifestationen finden sich an endokrinen Organen, Skelettsystemen, Augen, Ohren und Nieren. Schwerwiegende Spätfolgen stellen schließlich Infertilität, Sekundärmalignome und psychosoziale Erkrankungen dar.

\section{Fast jeder Dritte hat ein chronisches Gesundheitsproblem} Die Childhood Cancer Survival Study, eine retrospektive Kohortenstudie aus den USA, hat knapp 10400 Überlebende fünf Jahre nach Therapie mit ihren Geschwistern (3034) verglichen. 28\% der früheren Krebspatienten hatten mindestens ein chronisches Problem, das höchste Risiko hatten Überlebende nach Knochen- und Hirntumoren sowie nach Morbus Hodgkin.

Bei schwerwiegenden Spätfolgen wie Gelenkersatz, Herzinsuffizienz, Zweitmalignom, kognitiven Störungen, KHK,
Schlaganfall, Hörverlust oder ovarieller Dysfunktion übertraf das relative Risiko dieser Menschen das einer genetisch ähnlichen Kohorte erheblich.

$\mathrm{mb}$.

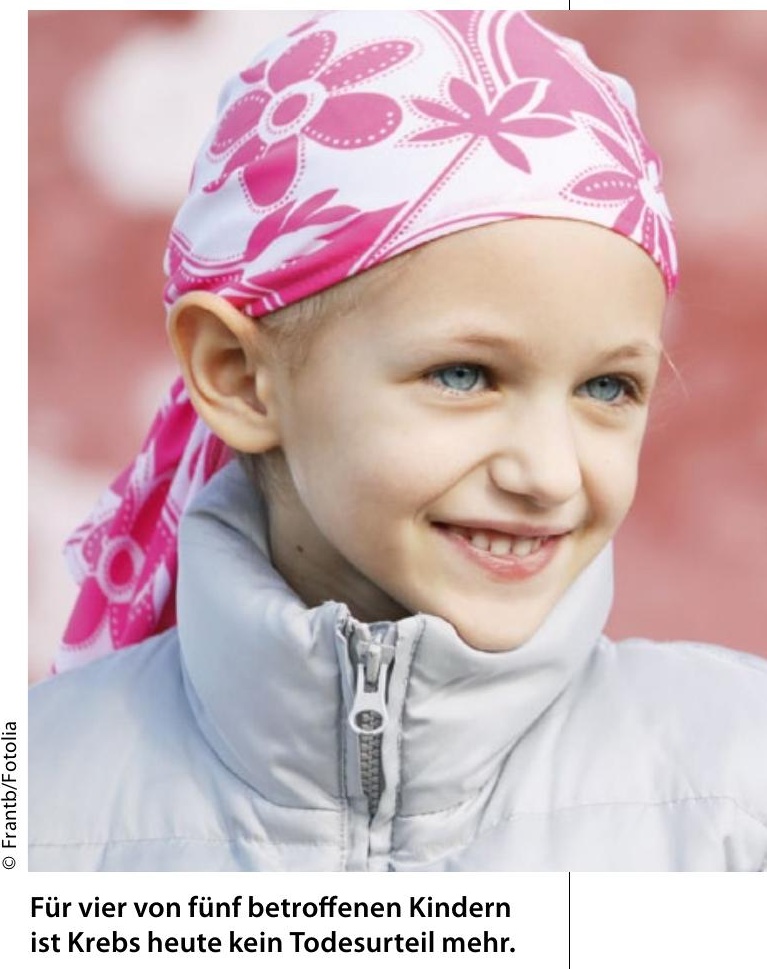

